\title{
Prefrontal compensatory engagement in TBI is due to altered functional engagement of existing networks and not functional reorganization
}

\author{
Gary R. Turner ${ }^{1,2,3 *}$, Anthony R. McIntosh ${ }^{3}$ and Brian Levine ${ }^{3,4,5}$ \\ 1 Centre for Stroke Recovery, Sunnybrook Health Sciences Centre, Heart and Stroke Foundation, Toronto, ON, Canada \\ 2 Department of Occupational and Rehabilitation Sciences, Faculty of Medicine, University of Toronto, Toronto, ON, Canada \\ ${ }^{3}$ Department of Psychology, University of Toronto, Toronto, ON, Canada \\ ${ }^{4}$ Department of Neurology, Faculty of Medicine, University of Toronto, Toronto, ON, Canada \\ ${ }^{5}$ Baycrest Center for Geriatric Care, Rotman Research Institute, Toronto, ON, Canada
}

\section{Edited by:}

Barry Horwitz, National Institutes of Health, USA

\section{Reviewed by:}

Marco Atzori, University of Texas at

Dallas, USA

Allison Carol Nugent, National Institute of Mental Health, USA

${ }^{*}$ Correspondence:

Gary R. Turner, Centre for Stroke Recovery, Sunnybrook Health Sciences Centre, Heart and Stroke Foundation, 2075 Bayview Avenue, Room A447, Toronto, ON M4N 3M5, Canada. e-mail: gary.turner@sunnybrook.ca
Functional neuroimaging studies of traumatic brain injury (TBI) have demonstrated altered neural recruitment, specifically within prefrontal cortex (PFC). This is manifest typically as increased recruitment of homologous regions of PFC (e.g., right ventrolateral PFC during performance of a verbal working memory task, possibly in response to damage involving the left PFC). The behavioral correlates of these functional changes are poorly understood. We used fMRI and multivariate analytic methods to investigate changes in spatially distributed activity patterns and their behavioral correlates in a sample of TBI patients with diffuse axonal injury (DAI, but without focal injury) and matched healthy controls. Participants performed working memory tasks with varying memory load and executive demand. We identified networks within left and right PFC that uniquely and positively correlated with performance in our control andTBI samples respectively, providing evidence of compensatory functional recruitment. Next we combined brain-behavior and functional connectivity analyses to investigate whether compensatory brain changes were facilitated by functional reorganization (i.e., recruitment of brain regions not engaged by our control sample) or altered functional engagement (i.e., differential recruitment of similar brain regions between the two groups based on task demands). In other words, does altered recruitment represent the instantiation of novel neural networks to support working memory performance after injury or the unmasking of extant, but behaviorally latent, functional connectivity? Our results support an altered functional engagement hypothesis. Areas within PFC that are normally coactivated during working memory are behaviorally relevant at an earlier stage of difficulty forTBI patients as compared to controls. This altered functional engagement, also evident in the aging literature, is attributable to distributed changes owing to significant DAI.

Keywords: traumatic brain injury, fMRI, working memory, functional connectivity, partial least squares, diffuse axonal injury

\section{INTRODUCTION}

Traumatic brain injury (TBI) is associated with altered patterns of neural recruitment during working memory (McAllister et al., 1999; Turner and Levine, 2008). This is typically manifest as increased activity in homologous regions of prefrontal cortex (PFC) or more spatially dispersed activity adjacent to areas implicated in non-injured controls (Christodoulou et al., 2001). Yet the cognitive implications of this altered recruitment are poorly understood. Altered functional brain response during working memory in TBI has been observed in the context of both poorer (e.g., Christodoulou et al., 2001) and equivalent task performance (e.g., Turner and Levine, 2008; Newsome et al., 2009). A recent review of functional neuroimaging studies in TBI reported similarly equivocal findings (Levine et al., 2006). To date, few studies have directly correlated brain activity and working memory performance. Perlstein et al. (2004) observed positive correlations between performance on an N-back working memory task and activity in left PFC in patients with mild and moderate TBI. Similar findings were also observed during $\mathrm{N}$-back performance in a sample of more severely injured subjects, however, positive correlations were limited to posterior brain regions (Newsome et al., 2007). Thus it remains an open question as to whether these brain changes are compensatory, deleterious, or incidental to task performance following TBI. While the presence of functional brain changes in the context of preserved behavior suggests that such changes are unlikely to be deleterious, these data do not directly address whether these changes are compensatory.

Traumatic brain injury is associated with widespread disruptions in cerebral microvasculature and metabolic changes (Povlishock and Katz, 2005), thus altered functional recruitment may reflect physiological or systemic brain changes that, while co-occurring with working memory processing, are incidental to behavioral 
performance. Functional brain changes may also be secondary to strategy differences between TBI and control subjects as has recently been demonstrated in healthy aging (Paxton et al., 2008). Previous studies have reported patterns of increased activity in homologous regions of lateral PFC or more spatially dispersed recruitment of adjacent brain regions in TBI relative to non-injured controls. A similar pattern of decreased lateralized response has also recently been observed in an electrophysiological investigation of sustained attention performance following TBI (Molteni et al., 2009). If these altered brain activity patterns are positively associated with task performance, this would provide evidence for compensatory functional brain changes. Here we used multivariate analysis (spatialtemporal partial least squares, PLS; McIntosh and Lobaugh, 2004) to identify brain-behavior correlations simultaneously across the whole-brain. The emphasis is on those brain regions that are correlated with task-behavior in healthy control subjects and whether these brain-behavior correlations are altered following TBI.

Characterizing the contribution of functional brain changes to behavioral performance following TBI has been hindered by heterogeneity in the severity of injury, chronicity, and neuropathology [e.g., focal lesions versus diffuse axonal injury (DAI)] as well as differences in task demand (Levine et al., 2006). Moreover, variability in behavioral performance is considered a hallmark of TBI (Stuss et al., 1989, 1994) and likely impedes detection of consistent brain-behavior correlations in this population. The current study directly investigates the relationship between altered functional brain response and behavioral performance during verbal working memory following TBI. To address the challenges identified in these earlier reports here we restricted recruitment to participants who had sustained a moderate to severe TBI but who had evidence of DAI on neuroradiological report but without evidence of focal brain damage (see Materials and Methods). In addition, the task paradigm varied load and executive demands (EDs) within working memory independently, allowing us to identify patterns of functional brain changes related to both maintenance and executive control processing separately.

We previously reported enhanced right PFC activity associated with increased ED within working memory following TBI (Turner and Levine, 2008). Here we examine whether functional changes in distributed brain regions following brain injury correlate with verbal working memory performance using behavioral PLS (bPLS) analyses (McIntosh et al., 2004). Unlike earlier studies that have correlated voxel-wise univariate changes in brain response with behavior, bPLS identifies whole-brain patterns of activity that are predictive of task-performance. Additionally, we examine whether functional connectivity amongst those network nodes demonstrating the most reliable brain and behavior correlations from the bPLS analysis are modulated by working memory task demands. Specifically, we ask whether functional brain changes observed in these participants reflect a pattern of altered functional engagement (i.e., recruitment of similar brain regions at lower levels of task demand than controls) or functional reorganization (i.e., recruitment of novel brain regions not engaged by controls). In other words, does the altered recruitment observed during working memory tasks following TBI represent the instantiation of novel neural networks to support working memory performance after injury or the unmasking of extant, but behaviorally latent, functional connectivity.

\section{MATERIALS AND METHODS PARTICIPANTS}

All subjects were part of the Toronto TBI study (Levine et al., 2008) and were recruited based upon consecutive admissions to a level 1 trauma center. All subjects were right-handed, native English speakers and were screened for previous neurologic injury, major medical conditions affecting cognition, history of psychiatric illness, and the use of medications affecting cognition. Further details with respect to the patient demographics, injury characteristics, and recruitment inclusion and exclusion criteria of the larger sample have been reported elsewhere (Levine et al., 2008). Demographic and injury characteristics for the subset of patients included in the present report have been described in Turner and Levine (2008) and are only briefly reviewed here. We recognize that variability is a hallmark of TBI and we have addressed this variability in several ways. First, all subjects had sustained a closed head injury as a result of a motor vehicle accident. All were in the chronic stage of recovery at the time of study participation. The injury severity profile of the group included moderate and severe TBI participants as determined by trauma Glasgow Coma Score (GCS, Teasdale and Jennett, 1974), loss of consciousness and extent of post-traumatic amnesia. All participants underwent extensive behavioral testing as part of the larger Toronto TBI Study and data are reported in the earlier paper (Turner and Levine, 2008; Table 1). Neuropsychologically, participants had preserved neuropsychological performance on standardized testing and good functional recovery (return to previous level of work or school). All TBI participants in the current study had evidence of DAI-related neuropathology (hemosiderin deposits) on neuroradiological report. Moreover, patients were excluded from participation if they had evidence of focal lesions greater than $3 \mathrm{~mm}$ in diameter based on high resolution structural MRI, resulting in a final sample of relatively "pure" DAI participants. Other exclusion criteria included previous head injury, significant psychiatric history, or evidence of current or recent alcohol and drug abuse. Following screening a total sample of eight TBI participants (six male) were recruited for the study. Twelve neurologically normal participants (eight males) were also recruited. TBI and control participants were matched on age $[t(18)=0.785$ $(p>0.05 ; \mathrm{NS})]$ and education $[t(18)=-1.99(p>0.05 ; \mathrm{NS})]$. All control participants were right-handed, native English speakers, and were screened for previous neurological injury, history of psychiatric illness, or drug use.

\section{BEHAVIORAL TASK}

In the present study we employed a modified version of the Alphaspan protocol described by Postle et al. (1999) and based on earlier work by Craik (1986). For each task trial, participants were required to study a letter set consisting of either three or five consonant letter strings (set size or "load" manipulation). For the ED manipulation, participants were asked to either hold the letter set in mind in it's original sequence ("MAINTAIN" condition) or to order the consonants alphabetically and maintain the new sequence over a brief delay period ("ALPHABETIZE" condition). At the end of the delay, a probe was presented consisting of a letter and an ordinal position (e.g., L-4? - "Was 'L' the fourth letter in the set?"). On Maintain trials, the probe referred to the ordinal position in the original letter set while on Alphabetize trials the probe referred to the letter position following 
Table 1 | Cluster maxima from the behavior PLS (bPLS) analysis for the two-group analysis (LV 1).

\begin{tabular}{|c|c|c|c|c|c|c|c|c|}
\hline Lag & $x$ & $y$ & $z$ & Lat. & Anat. & BA & BSR & Clust. \\
\hline 0 & -4 & 21 & 36 & $L$ & GFd & 32 & 5.71 & 11 \\
\hline 0 & 40 & 10 & 40 & $\mathrm{R}$ & GFm & 9 & 6.25 & 13 \\
\hline 0 & -20 & -82 & -13 & $L$ & $\mathrm{GL}$ & 18 & 8.56 & 119 \\
\hline 0 & -8 & -46 & 6 & $L$ & CG & 29 & 7.40 & 78 \\
\hline 0 & 0 & -21 & -36 & & B. stem & & 6.34 & 25 \\
\hline 0 & -8 & -29 & -29 & $L$ & B. stem & & 4.91 & 22 \\
\hline 0 & 16 & -33 & -35 & $\mathrm{R}$ & B. stem & & 4.64 & 13 \\
\hline 0 & 16 & -59 & -14 & $\mathrm{R}$ & Cereb & & 6.05 & 24 \\
\hline 0 & 4 & -83 & -26 & $\mathrm{R}$ & Cereb & & 7.11 & 80 \\
\hline 1 & 8 & 52 & 27 & $\mathrm{R}$ & GFs & 9 & 5.86 & 11 \\
\hline 1 & -20 & 22 & 58 & $\mathrm{~L}$ & GFs & 6 & 5.90 & 14 \\
\hline 1 & -51 & -36 & 53 & $\mathrm{~L}$ & LPi & 40 & 5.28 & 11 \\
\hline 1 & -28 & -52 & 54 & $\mathrm{~L}$ & LPs/LPi & $7 / 40$ & 9.53 & 18 \\
\hline 1 & -24 & -82 & -13 & $\mathrm{~L}$ & GF & 18 & 9.02 & 143 \\
\hline 1 & -44 & -85 & 15 & $\mathrm{~L}$ & $\mathrm{GOm}$ & 19 & 7.06 & 11 \\
\hline 1 & 20 & -86 & -13 & $\mathrm{R}$ & $\mathrm{GL}$ & 18 & 12.10 & 66 \\
\hline 1 & -8 & 21 & 32 & $\mathrm{~L}$ & $\mathrm{GC}$ & 32 & 5.66 & 22 \\
\hline 1 & -4 & -50 & 6 & $L$ & CG & 30 & 6.34 & 20 \\
\hline 1 & 0 & -25 & -32 & & B. stem & & 6.65 & 62 \\
\hline 1 & 24 & -63 & -14 & $\mathrm{R}$ & Cereb & & 6.10 & 28 \\
\hline 2 & 24 & 52 & 38 & $\mathrm{R}$ & GFs & 8 & 5.54 & 15 \\
\hline 2 & -48 & 51 & 12 & $L$ & GFm & $10 / 46$ & 5.82 & 16 \\
\hline 2 & 44 & 47 & 16 & $\mathrm{R}$ & GFm & 46 & 13.75 & 38 \\
\hline 2 & 40 & 16 & 18 & $\mathrm{R}$ & $\mathrm{GFi}$ & $44 / 45$ & 5.91 & 25 \\
\hline 2 & -20 & 11 & 62 & $L$ & GFs & 6 & 6.04 & 13 \\
\hline 2 & -32 & -51 & -14 & $\mathrm{~L}$ & GF & 37 & 6.42 & 24 \\
\hline 2 & 48 & 18 & -21 & $\mathrm{R}$ & GTs & 38 & 6.22 & 13 \\
\hline 2 & -24 & -53 & 21 & $\mathrm{~L}$ & GTm & 39 & 5.61 & 22 \\
\hline 2 & 16 & -39 & 76 & $\mathrm{R}$ & LPC & 7 & 7.90 & 14 \\
\hline 2 & -16 & -64 & 44 & $L$ & $\mathrm{PCu}$ & 7 & 7.56 & 82 \\
\hline
\end{tabular}

Table 1 | Continued

\begin{tabular}{lcrrlllll}
\hline Lag & $\boldsymbol{x}$ & $\boldsymbol{y}$ & $\boldsymbol{z}$ & Lat. & Anat. & BA & BSR & Clust. \\
\hline & & & & & & & & \\
2 & 24 & -86 & -13 & $\mathrm{R}$ & GF & 18 & 10.65 & 51 \\
2 & -44 & -88 & 23 & $\mathrm{~L}$ & GO & 19 & 5.86 & 11 \\
2 & -44 & -90 & -9 & $\mathrm{~L}$ & GOi & 18 & 7.67 & 53 \\
2 & 0 & -46 & 13 & & GC & 30 & 6.97 & 41 \\
2 & -20 & -25 & -39 & $\mathrm{~L}$ & B. stem & & 6.58 & 81 \\
2 & 28 & -63 & -17 & $\mathrm{R}$ & Cereb & & 6.64 & 22 \\
3 & 40 & 47 & 16 & $\mathrm{R}$ & GFm & 46 & 9.90 & 18 \\
3 & 28 & -46 & 21 & $\mathrm{R}$ & GTm & 39 & 5.74 & 18 \\
3 & -28 & -50 & 14 & $\mathrm{~L}$ & GTm & 39 & 5.75 & 19 \\
3 & -24 & -59 & 58 & $\mathrm{~L}$ & PCu & 7 & 6.31 & 69 \\
3 & 24 & -63 & 58 & $\mathrm{R}$ & PCu & 7 & 5.77 & 12 \\
3 & 24 & -90 & -16 & $\mathrm{R}$ & GF & 18 & 9.49 & 54 \\
3 & 16 & -33 & -39 & $\mathrm{R}$ & B. stem & & 7.32 & 11 \\
3 & 32 & -55 & -21 & $\mathrm{R}$ & Cereb & & 5.72 & 27 \\
4 & -16 & -83 & -26 & $\mathrm{~L}$ & Cereb & & 5.18 & 11 \\
4 & 20 & -86 & -19 & $\mathrm{R}$ & Cereb & & 5.70 & 24 \\
\hline
\end{tabular}

This collection of brain regions demonstrated a positive correlation with task accuracy differentially across group and condition indicated in Figure 1. Cluster thresholds (i.e., how reliably each brain region expressed the LV) was determined using a bootstrapping procedure (see Materials and Methods). Here we report $B S R^{\prime} s>4.0$ (corresponding approximately to $p<0.0001$ ) with a minimum spatial extent of 10 voxels. Lag refers to the temporal window with lag 0 corresponding to 2-4 s after stimulus onset. Each lag represents a 2-s time window (see Materials and Methods for details). Voxel coordinates are reported in Talairach coordinates.

Lat, laterality; Anat., anatomical region; abbreviations consistent with the atlas (see below). BA, Brodmann area; BSR, bootstrap ratio; Clust., cluster size (in voxels); GFd, medial frontal gyrus; GFm, middle frontal gyrus; GL, lingual gyrus; CG, cingulate gyrus; B. stem, brain stem; Cereb, cerebellum; GFs, superior frontal gyrus; LPs, superior parietal lobule; LPi, inferior parietal lobule; GF, fusiform gyrus; GOm, middle occipital gyrus; GFi, inferior frontal gyrus; GTs, superior temporal gyrus; GTm, middle temporal gyrus; $L P C$, paracentral lobule; $P C u$, precuneus; GO, orbital frontal gyrus; GOi, inferior occipital gyrus.. alphabetization of the list. Probability of a correct probe was set at 0.5 for all trials in all conditions. Prior to scanning, each participant completed a training session consisting of step-by-step instructions for each task condition. Once there were no further questions for the administrator, all subjects completed 20 further trials (five trials in each of the four conditions) immediately prior to entering the MR scanner. During scanning, participants completed 28 trials of each of the four task conditions (Alphabetize three- and five-letter sets, Maintain three- and five-letter sets) during a single scanning session. Within each session a total of four individual scans were acquired. Trials were grouped by ED with two blocks of seven trials at each level of ED presented during a single scan acquisition. Total stimulus onset asynchrony was $18000 \mathrm{~ms}$ (three-letter trials) or $19000 \mathrm{~ms}$ (five-letter trials). Each individual scan acquisition was $12 \mathrm{~min}$ in duration (see Turner and Levine, 2008 for a full trial schematic).

\section{fMRI SCANNING AND ANALYSES}

Scanning was performed at Sunnybrook Health Sciences Centre on a research-dedicated whole-body 3.0 T MRI system (Signa 3T94 hardware, VH3M3 software; General Electric Healthcare, Waukesha, WI, USA) with a standard quadrature bird-cage head coil. Participants were placed in the scanner in supine position, with their head firmly placed in a vacuum pillow to minimize head movement. Earplugs were provided to reduce the noise from the scanner. A volumetric anatomical MRI was performed before functional scanning, using standard high-resolution 3D T1-weighted fast spoiled gradient echo $(\mathrm{FSPGR})$ images $(\mathrm{TR} / \mathrm{TE}=7.2 / 3.1 \mathrm{~ms}$, inversionrecovery prepared $\mathrm{T} 1=300 \mathrm{~ms}$, flip angle $15^{\circ}, 256 \times 192$ acquisition matrix, 124 axial slices $1.4 \mathrm{~mm}$ thick, voxel size $=0.86 \mathrm{~cm} \times 0.86 \mathrm{~cm}$, FOV $=22 \mathrm{~cm} \times 16.5 \mathrm{~cm}$ ). Functional imaging was performed to measure the blood oxygenation level dependent (BOLD) effect (Ogawa et al., 1990). Scans were obtained using a single-shot T2*weighted pulse with spiral in-out, achieving 26 slices, each $5 \mathrm{~mm}$ thick $\left(\mathrm{TR} / \mathrm{TE}=2000 / 30 \mathrm{~ms}\right.$, flip angle $70^{\circ}, 64 \times 64$ acquisition matrix, 26 axial slices $5 \mathrm{~mm}$ thick, voxel size $=3.125 \mathrm{~mm} \times 3.125 \mathrm{~mm}$, slice spacing $=0$, FOV $=20 \mathrm{~cm} \times 20 \mathrm{~cm}$ ). Data pre-processing was performed using Analysis of Functional NeuroImages software (Cox and Hyde, 1997). At the beginning of each scan, stimulus presentation was synchronized with the start of image acquisition through a triggering pulse input from the scanner to the E-prime presentation software. The initial 10 time points from each functional image volume were excluded from the analyses to allow for stabilization 
of the magnetic field gradients. Time-series data were spatially coregistered (aligned volumetrically to a reference image within the run, using the 3 dvolreg program in AFNI) to correct for small head motion using a 3-D Fourier transform interpolation, and the linear trends were removed. Uncorrected head motion (spikes) was identified through visual inspection and reduced through averaging the two surrounding time points. Physiological motion (respiration and heart beat) was also removed through linear filtering. Finally, slice timing correction was carried out to account for the time dependent discrepancy between the initial and final slice acquisitions. Images were then spatially transformed to an fMRI spiral scan template generated from 30 subjects scanned locally. This template was registered to the MNI305 template. The transformation of each subject to the spiral template was achieved using a 12-parameter affine transform with sinc interpolation as implemented in SPM99 (http://www.fil. ion.ucl.ac.uk/spm; Friston et al., 1995). Images were smoothed with an 8-mm isotropic Gaussian filter before analysis. For each subject, "brain" voxels in a specific image were defined as voxels with an intensity greater than $15 \%$ of the maximum value in that image. The union of masks was used for group analyses as described below.

\section{PARTIAL LEAST SQUARES ANALYSES}

Primary fMRI data analysis was carried out using spatiotemporal PLS (McIntosh et al., 1996; and for a full review of the method see McIntosh et al., 2004). In brief, PLS computes an optimal least squares fit to the "cross-block" correlation between the exogenous and dependent measures. Limiting the analysis to this part of a correlation or covariance matrix distinguishes PLS from principal components analysis (PCA) as solutions are constrained to the part of the covariance structure that is attributable to experimental manipulations or related to behavior. PLS is particularly suitable for neuroimaging data where measures are highly correlated as items within a block are not adjusted for these correlations. PLS operates on the entire data structure at once with one data matrix for each group. Within group, condition blocks are stacked, and each subject has a row of data within each block. With $\mathrm{n}$ subjects and $\mathrm{k}$ conditions, there are $n b \times k$ rows in the group matrix. Columns contain the hemodynamic response (HRF) signal intensity measure at each voxel at each time point. To account for the lag in hemodynamic response, the lag window is $6(\mathrm{TR}=2 ; 12 \mathrm{~s})$, beginning at trial onset -1 TR. The HRF for each trial is expressed as the intensity difference from trial onset. The first column has intensity for the first voxel at the first time point, the second column has the intensity for the first voxel at the second time point etc. With $m$ voxels and $t$ time points, there are $m \times t$ columns in the matrix.

Singular value decomposition (SVD) is applied to re-express the matrix as a set of orthogonal singular vectors or latent variables (LVs). LVs are analogous to eigenvectors in PCA and account for the matrix in decreasing order of magnitude. Each LV contains a pair of vectors relating brain activity and experimental design. Singular values are used to calculate the proportion of the matrix accounted for by each LV. Statistical assessment of the LVs is done using permutation tests (500 permutations) and bootstrap estimation (100 estimations) of standard errors for the voxel saliences. The permutation test assesses whether the effect represented in a given LV is sufficiently strong, in a statistical sense, to be different from random noise. Permutations were carried out using sampling without replacement to reassign the order of conditions for each subject. PLS is recalculated for each new sample, and the probability of the permuted exceeding observed is determined. To determine the stability of the voxel saliences for the LVs retained, bootstrap estimates are calculated using sampling with replacement and PLS is rerun keeping the assignment of experimental conditions fixed for all subjects. No corrections for multiple comparisons are necessary because the voxel saliences are calculated in a single mathematical step on the whole brain. The ratio of the salience to the bootstrap standard error is approximately equivalent to a $z$-score and is used assess the reliability of the individual voxel saliences. Two forms of PLS were performed. The first, bPLS, was the primary analysis to investigate group differences in brain-behavior correlations for the working memory tasks. bPLS is a variant of PLS that identifies LVs that capture task- and group-dependent patterns of brain-behavior correlations (McIntosh et al., 2004). The correlation of behavior measures and the fMRI signal is computed across subjects within each task, producing within-task brain-behavior correlations for each of the four task conditions and groups. The strength and reliability of the LVs for bPLS is carried out as described above. For these analyses an LV was considered "significant" if the probability of the observed singular value for the LV given permutation testing was less than 0.01 . Effects were considered to be reliable if the ratio of observed to estimated effects on bootstrap testing was greater than 4 . This corresponds to a probability of approximately $p<0.0001$ assuming bootstrap estimates were normally distributed (Figure 1; Table 1). This addressed the fundamental question as to whether those regions correlating most strongly with behavior differed between our two groups. From this analysis we identified seed regions of interest (ROI) to be used in the combined behavior and functional connectivity analysis.

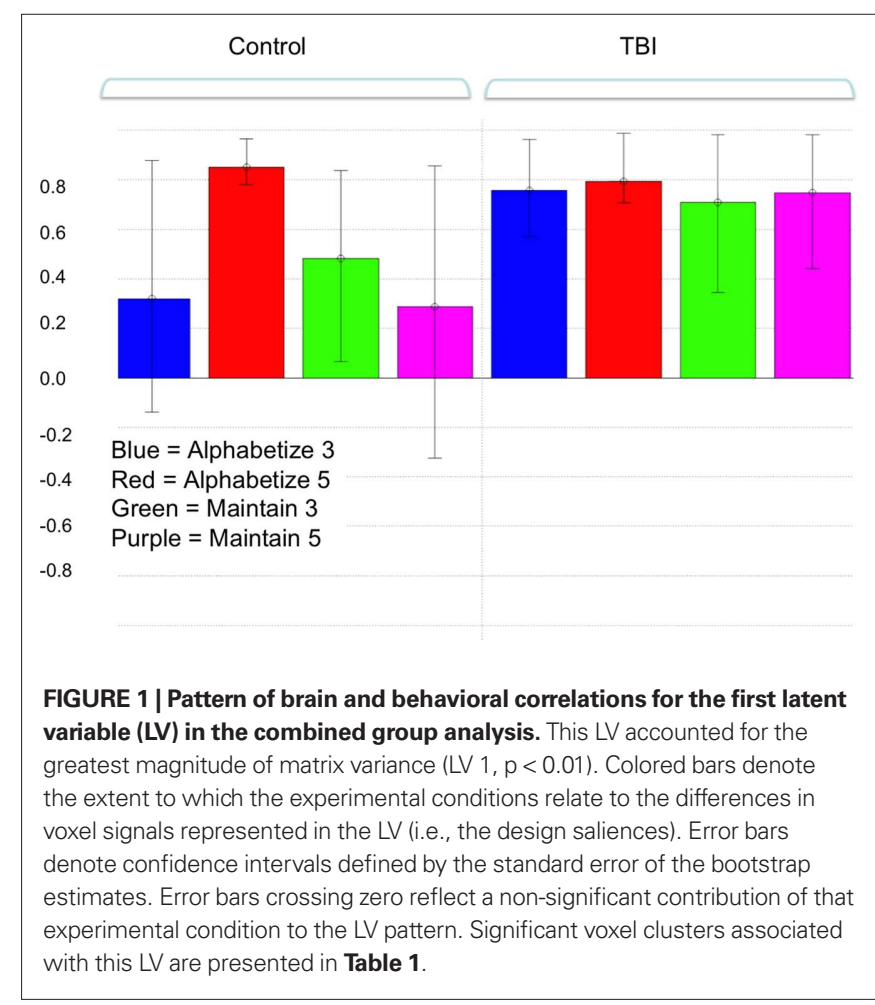


This second analysis used a behavior-seed PLS (sPLS) technique (McIntosh et al., 2004) to address the question of whether the behavioral relevance of regions demonstrating reliable brainbehavior correlations would depend upon their functional connectivity to other brain regions and whether these connectivity patterns varied between our two groups. sPLS identifies LVs that capture task- and group-dependent changes in functional connectivity between the seed ROI and the rest of the brain (i.e., brainseed correlations). The correlation of the fMRI signal for the seed and for the rest of the brain is computed across subjects within groups within each task, resulting in a matrix of within group and within-task brain-seed correlation maps. SVD of the brain-seed correlation matrix produces three new matrices: the singular image of voxel saliences, singular values, and task saliences. The variation across the task saliences indicates whether a given LV represents a similarity or difference in the brain-seed correlation across tasks. This can also be shown by calculation of correlation between the brain scores (dot-product of the voxel salience and fMRI data) and seed fMRI signal for each task. The voxel saliences give the corresponding spatiotemporal activity pattern. Statistical assessment is identical to that used for bPLS.

\section{RESULTS \\ BEHAVIOR}

Full behavioral analyses are presented in Turner and Levine (2008), and only the between group results relevant to the current study are described here. As we were investigating brain and behavioral correlates at different levels of working memory task demand, task accuracy provided the best assay as it is less susceptible than reaction time measures to processing demands unrelated to working memory (e.g., scanning, retrieval, motor speed). One TBI patient was identified as an outlier for accuracy (TBI \# 8). Trial-wise analysis for this patient revealed a consistent pattern of timing out on Alphabetize trials, greater for five- than for three-letter sets sizes. On those trials where responses were recorded, performance was within the average range of the TBI group for both three- and fiveletter sets (78 and 67\% respectively). This pattern is indicative of a task-specific decrement at response as opposed to disengagement or poor arousal. As we were interested in neural response during encoding and delay trial epochs, and not at probe, we did not exclude this patient's data. For the purposes of statistically assessing group effects on task performance, we used a winsorizing procedure by which the outlier values for this subject's accuracy on Alphabetize $3 / 5$ trials were trimmed to 2 SD below the true mean of the sample (i.e., exclusive of the outlier). To confirm that our results were not unduly influenced by this outlier correction we ran all analyses with this subject's original data. There was a marginal but insignificant impact of the correction on the robustness of the bPLS output (see bPLS Results below). There was no impact on the behavioral results. Statistical analysis was carried out using a two group (control versus $\mathrm{TBI}) \times 2(\mathrm{ED}) \times 2($ set size $)$ repeated measures ANOVA. There was a significant main effect of ED and set size (Set) $[F(1)=18.51$ (ED); $F(1)=11.42$ (Set), $p<0.01$ for both comparisons] with poorer performance observed during the Alphabetize and set size five conditions. There was no main effect of group $[F(1)=2.54$; NS] and no group $\times \operatorname{ED}[F(1)=0.094, N S]$ or group $\times \operatorname{Set}[F(1)=0.182$, $\mathrm{NS}$ ] interactions, indicating that the main effects of condition were stable across both the control and TBI groups. Post hoc analyses revealed that there were no group differences on any of the tasks ( $p>0.05$, all comparisons).

\section{fMRI}

\section{Behavior PLS (brain and behavior)}

A two-group bPLS with accuracy as the behavioral measure identified one significant LV $(p<0.01)$ reflecting brain and behavior correlations for all tasks in the TBI group but only for the Alphabetize 5 task in controls ${ }^{1}$ (Figure 1). Positive saliences for this LV (Table 1) were observed in areas commonly implicated in neuroimaging studies of working memory (Wager and Smith, 2003). These task-related differences in brain-behavior correlations between our two groups suggests that TBI subjects were engaging this network, which included lateral aspects of PFC bilaterally, to support behavioral performance at lower levels of task demand than controls. That is, whereas controls engaged the network at the highest level of ED, Alphabetize 5, TBI patients did so at all levels.

We next conducted bPLS analyses for the control and TBI groups separately. For the control group analysis, one significant LV emerged $(\mathrm{p}<0.01)$. This LV reflected positive brain and behavior correlations for all tasks (Figure 2A; Table 2). Brain regions demonstrating the highest salience for this LV included two regions of left lateral PFC [inferior frontal gyrus (GFi) and middle frontal gyrus $(\mathrm{GFm})]$ as well as in posterior parietal and visual association cortices. For the TBI group, one LV approached statistical significance $(\mathrm{p}<0.058)$. As with the control group, this LV reflected positive brain and behavior correlations for all tasks. However, in contrast to the control data, the highest salience for this LV occurred in regions of right lateral PFC (Figure 2B; Table 3) in addition to posterior parietal regions. These data extend the results of the combined group analysis and highlight the role of right lateral PFC in supporting working memory performance following TBI, particularly at lower levels of task demand.

\section{Seed PLS (brain, behavior, and functional connectivity)}

The results of the bPLS analyses supported our prediction that right frontal recruitment during working memory performance was compensatory for TBI participants. Next we asked whether this right PFC activity represented the instantiation of novel PFC networks to support working memory performance (functional reorganization) or the engagement of existing functional connections to support performance as load or executive control demands within working memory increase (altered functional engagement). For this analysis, we entered the four cluster maxima within PFC from the bPLS results (see Figure 2, i-iv; Tables 2 and 3) into a combined behavior and functional connectivity PLS analysis (Grady et al., 2003). Specifically, we investigated within a single PLS analysis: (i) how these regions functionally connect with other brain areas, in particular the other frontal ROIs, and how connectivity is altered by task demand (sPLS) (ii) whether these patterns of functional connectivity were related to task performance (bPLS)

${ }^{1}$ The brain and behavior correlation $(\mathrm{r}=0.48)$ was also reliable for M3 in controls based on bootstrap estimates of standard error. However, this likely reflects near-ceiling performance on this task $[$ mean $=0.96(0.03)]$ and is not discussed further here. 


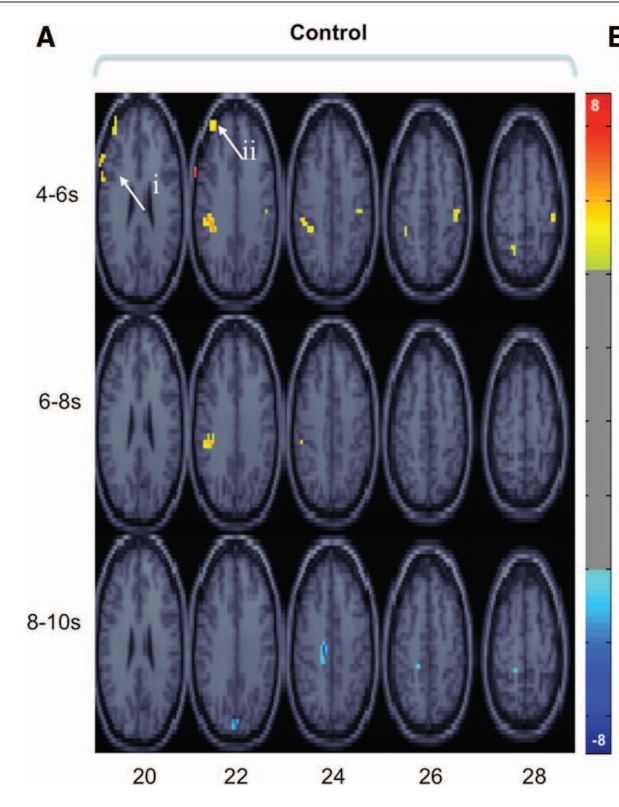

B TB

FIGURE 2 | Brain regions showing significant salience for the first LV in each group-wise bPLS analyses [i.e., those brain regions demonstrating positive correlations between brain response and task accuracy in control (A) and TBI (B) groups for all tasks]. Voxel salience reliabilities were determined by bootstrap ratios (BSR; range is indicated by the color bar). For these images BSR was set at $4\left(p<10^{-4}\right.$, corresponding approximately to a $p<0.0001$ ) and cluster size was $>10$ voxels. Roman numerals indicate PFC

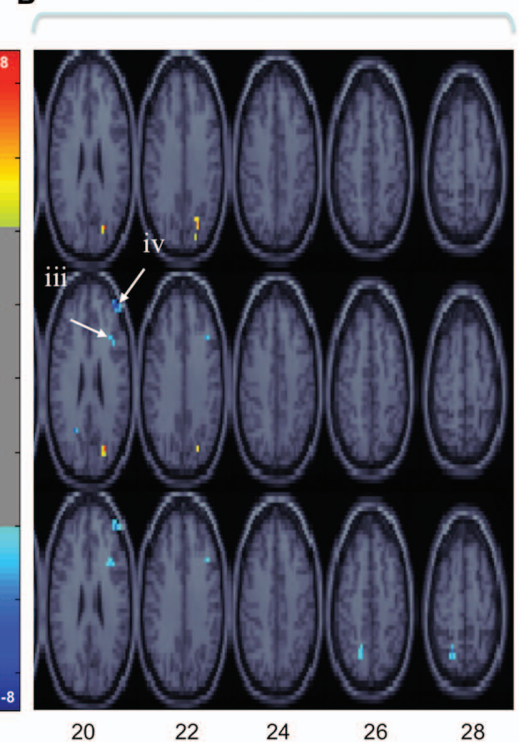

regions with the most reliable and positive brain and behavior correlations across all tasks. Talairach coordinates $(x, y, z)$ of cluster maxima: (i) left inferior frontal gyrus (GFi: -67, 9, 29); (ii) left middle frontal gyri (GFm: -40, 48, 23); (iii) right posterior middle frontal gyrus (pGFm: 36, 21, 25); (iv) right anterior middle frontal gyrus (aGFm: 40,47,16). These voxels are used as seed regions in subsequent seed and behavior PLS analyses (see text and Figures 3 and 4).

reflecting this pattern included bilateral anterior GFm (aGFm) (BA 46/10), left posterior cingulate gyrus, left precuneus (BA 7), and left posterior middle temporal gyrus (BA 39; Figure 3Aiii).

\section{Right anterior middle frontal gyrus (BA 46/10)}

Latent variable $1(p<0.001)$ demonstrated reliable and positive behavior and seed correlations for both groups. For the control group, positive correlations with behavior and activity in the seed region were reliable for Alphabetize 5 and, to a lesser extent, the Maintain 3 task (see note 1 above). In contrast, this pattern was reliable for both Alphabetize 3 and 5 as well as the Maintain 5 tasks in the TBI group (Figure 3Aiv). Positive saliences were observed in right posterior GFm (pGFm), left aGFm, posterior cingulate gyrus, left superior parietal lobule and the inferior occipital cortex and temporal-occipital junction bilaterally (Figure 3Aiv).

In sum, these results suggest a differential pattern of stepwise functional recruitment of right $\mathrm{PFC}$ regions between our two groups as working memory task demands increased. It is important to note here that the seed voxels selected for the functional connectivity analyses, derived from the bPLS results, were different for our two groups (Figure 2; Tables 2 and 3). We cannot rule out that the differential patterns of functional connectivity reported here may be, in part, attributable to the selection of these different seed ROIs for our two groups. Critically, however, the group differences in functional connectivity were task-dependent - and this pattern is not compatible with a biased-selection account. The pattern of task-dependent changes in functional connectivity between the Control and TBI groups is illustrated in Figure 3B. Bilateral functional connectivity within PFC is evident during working memory performance 
Table 2 | Cluster maxima from the behavior PLS (bPLS) analysis for the control group (LV 1, see Figure 2A).

\begin{tabular}{|c|c|c|c|c|c|c|c|c|c|}
\hline Lag & $x$ & $y$ & $z$ & Lat. & Anat. & BA & BSR & Clust. & ROI \\
\hline 0 & -44 & 44 & 16 & $\mathrm{~L}$ & GFm & 46 & 5.22 & 12 & \\
\hline 0 & 28 & 6 & 44 & $\mathrm{R}$ & GFm & 6 & 6.17 & 12 & \\
\hline 0 & 24 & -67 & -13 & $\mathrm{R}$ & GF & 19 & 7.16 & 54 & \\
\hline 0 & -36 & -78 & -10 & $L$ & GOm & 19 & 7.89 & 58 & \\
\hline 1 & -40 & 48 & 23 & $L$ & GFm & $9 / 46$ & 6.60 & 22 & ii \\
\hline 1 & -67 & 9 & 29 & $L$ & GFi & $44 / 6$ & 8.06 & 20 & i \\
\hline 1 & 28 & 6 & 48 & $\mathrm{R}$ & GFm & 6 & 5.44 & 11 & \\
\hline 1 & -63 & -46 & 10 & $\mathrm{~L}$ & GTs & 22 & 6.34 & 18 & \\
\hline 1 & 44 & -28 & 53 & $\mathrm{R}$ & LPi & 40 & 5.27 & 30 & \\
\hline 1 & -51 & -30 & 31 & $\mathrm{~L}$ & Gsm & 40 & 6.67 & 44 & \\
\hline 1 & 32 & -48 & 50 & $\mathrm{R}$ & LPs & 7 & 4.84 & 12 & \\
\hline 1 & -24 & -55 & 58 & $L$ & LPS & 7 & 5.42 & 27 & \\
\hline 1 & -24 & -78 & -13 & L & $\mathrm{GF} / \mathrm{GL}$ & 18 & 7.49 & 108 & \\
\hline 1 & 28 & -78 & -13 & $\mathrm{R}$ & $\mathrm{GF} / \mathrm{GL}$ & 18 & 6.44 & 75 & \\
\hline 2 & -44 & 26 & -18 & $L$ & GTs & 38 & 6.78 & 21 & \\
\hline 2 & -51 & -29 & 35 & L & LPi & 40 & 6.00 & 24 & \\
\hline 2 & 32 & -56 & 54 & $\mathrm{R}$ & LPs & 7 & 6.10 & 15 & \\
\hline 2 & -24 & -59 & 62 & $\mathrm{~L}$ & $\mathrm{PCu}$ & 7 & 8.14 & 17 & \\
\hline 2 & 8 & -63 & 66 & $\mathrm{R}$ & $\mathrm{PCu}$ & 7 & 5.21 & 11 & \\
\hline 2 & -48 & -63 & -10 & L & $\mathrm{GOm}$ & $37 / 19$ & 6.17 & 28 & \\
\hline 3 & -40 & -66 & -3 & L & $\mathrm{GOm}$ & 19 & 6.33 & 11 & \\
\hline 2 & -44 & -84 & 23 & L & $\mathrm{GO}$ & 19 & 6.44 & 26 & \\
\hline 2 & 28 & -90 & -16 & $\mathrm{R}$ & GF & 18 & 6.81 & 91 & \\
\hline 2 & -8 & -98 & -9 & L & $\mathrm{GL}$ & 18 & 6.07 & 11 & \\
\hline 3 & 48 & -64 & -27 & $\mathrm{R}$ & Cereb & & 7.44 & 58 & \\
\hline
\end{tabular}

These regions demonstrated a positive correlation with task accuracy. Cluster threshold (BSR) was $>4$, corresponding approximately to $p<0.0001$, with minimum size of 10 voxels. All column headings and abbreviations as in Table 1.

ROI, region of interest (roman numerals correspond to regions highlighted in Figure 2).

whenever load is increased or when EDs are present. However, the threshold at which this expanded right PFC network is functionally necessary for working memory performance is reduced following TBI, consistent with an altered functional engagement hypothesis.

\section{DISCUSSION}

Traumatic brain injury is associated with altered functional recruitment during the performance of cognitively demanding tasks. We have recently demonstrated this in the context of working memory performance using fMRI methods (Turner and Levine, 2008), These functional brain changes have been reported in other cognitive domains (e.g., sustained attention; Dockree et al., 2004; and see Levine et al., 2006 for a review of functional neuroimaging and TBI) and with other imaging modalities (e.g., electrophysiology; Dockree et al., 2004; Molteni et al., 2009; see Duncan et al., 2005 for a review). However, the behavioral implications of this altered neural activity have been poorly characterized. Here we investigated whether altered patterns of neural recruitment observed following TBI are compensatory and, if so, do they represent the instantiation of novel neural networks (i.e., true functional reorganiza- tion) or enervation of functional networks that are behaviorally latent in the undamaged or under-challenged brain (i.e., altered functional engagement).

In the first analysis, we used bPLS to investigate whether the altered functional recruitment of brain regions observed during working memory performance was related to task accuracy. We hypothesized, based on previous reports from the functional neuroimaging literature (e.g., McAllister et al., 1999; Perlstein et al., 2004), that over-recruitment of areas within right PFC which we had reported previously in this TBI sample would be positively correlated with task accuracy (i.e., compensatory). These data provide strong support for our hypothesis. Activity in a right lateralized network, including two distinct regions of right lateral PFC in the vicinity of the aGFm on the border of BA 10/46 and a more dorsal region of GFm on the border of BA 44/45 was correlated with working memory task accuracy in our TBI group (Figure 2B; Table 3). Similar regions of right lateral PFC were observed by Perlstein et al. (2004) to track positively with working memory load in their TBI sample. This region has also been implicated in supra-span working memory performance in non-injured participants (e.g., Rypma et al., 1999) and during working memory performance in healthy aging (Erickson et al., 2007). However, these data represent the first time that activity in this region has been directly and positively correlated with performance on a working memory task, specifically taxing executive control processes, in a TBI sample.

In contrast to the right lateralized brain and behavior correlations observed in our TBI sample, task accuracy in the control sample during all tasks was predominantly associated with activity in a network of left lateralized brain regions, including GFi and GFm (BA 46/44; Figure 2A; Table 2), although this network expanded to include right lateral PFC during Alphabetize 5 trials (Figure 1; Table 1). This pattern replicates many previous reports implicating lateral PFC in working memory tasks involving a significant executive control component (Curtis and D'Esposito, 2003; see Cabeza and Nyberg, 2000 for a review). These results provide strong support for our original hypothesis that recruitment of right lateral PFC during cognitively demanding tasks, as has now been reported in several studies, is compensatory. Moreover, a similar finding from our laboratory using a feature integration task suggests that this pattern of compensatory neural recruitment following TBI is not specific to working memory but may represent a domain-general response to increased cognitive challenge.

Few reports to date have directly correlated brain and behavior measures in a TBI sample. Our findings are consistent with those of McAllister et al. (1999) who observed positive correlations between activity in left GFi and accuracy on a working memory task in patients with mild TBI; a pattern we also observed in our healthy controls (they did not report group-specific correlations). However, our data are not consistent with those of Newsome et al. (2007) who failed to observe significant correlations between brain response and performance during the 2-back condition of an N-back working memory task. This task is similar to that used by McAllister et al. (1999), where maximal brain response during the 2-back condition was observed in their sample of mild TBI patients. We suggest that patient heterogeneity in the sample of Newsome et al. (2007) may have limited their ability to detect 
Table 3 | Cluster maxima from the behavior PLS (bPLS) analysis for the TBI group (see Figure 2B).

\begin{tabular}{lrrrllllll}
\hline Lag & $\boldsymbol{x}$ & $\boldsymbol{y}$ & $\boldsymbol{z}$ & Lat. & Anat. & BA & BSR & Clust. & ROI \\
\hline 0 & -20 & -82 & -13 & $\mathrm{~L}$ & GL & 18 & -6.25 & 12 & \\
0 & 8 & -82 & -13 & $\mathrm{R}$ & GL & $17 / 18$ & -5.80 & 18 & \\
0 & 0 & -35 & 2 & $\mathrm{R}$ & Pulvinar & & -5.47 & 24 & \\
0 & -20 & -25 & -29 & $\mathrm{~L}$ & B. stem & & -6.98 & 15 & \\
1 & 20 & -86 & -13 & $\mathrm{R}$ & GL/GF & 18 & -7.19 & 22 & \\
1 & 0 & -25 & -32 & & B. stem & & -10.13 & 16 & \\
1 & -20 & -86 & -19 & $\mathrm{~L}$ & Cereb & & -6.47 & 19 & \\
2 & 40 & 47 & 16 & $\mathrm{R}$ & GFm & 46 & -11.98 & 28 & iii \\
2 & 36 & 21 & 25 & $\mathrm{R}$ & GFm/GFi & $46 / 44$ & -7.89 & 12 & iv \\
2 & -28 & -57 & 25 & $\mathrm{~L}$ & GTm & 39 & -7.69 & 14 & \\
2 & 24 & -86 & -13 & $\mathrm{R}$ & GF & 18 & -6.41 & 13 & \\
2 & -20 & -29 & -36 & $\mathrm{~L}$ & B. stem & & -7.56 & 16 & \\
2 & 12 & -29 & -39 & $\mathrm{R}$ & B. stem & & -6.25 & 19 & \\
3 & 44 & 47 & 16 & $\mathrm{R}$ & GFm & 46 & -9.99 & 16 & iii \\
3 & 36 & 21 & 25 & $\mathrm{R}$ & GFm/GFi & $46 / 44$ & -6.01 & 13 & iv \\
3 & -28 & -50 & 17 & $\mathrm{~L}$ & GTm & 39 & -5.08 & 14 & \\
3 & -24 & -56 & 54 & $\mathrm{~L}$ & LPs & 7 & -7.13 & 18 & \\
4 & -16 & -83 & -26 & & Cereb & & -5.23 & 14 & \\
\hline & & & & & & & & & \\
\hline
\end{tabular}

Cluster thresholding criteria as in Table 2. All abbreviations as in Tables 1 and 2.

significant correlations. While other reports examining functional brain changes during working memory performance following TBI did not report direct brain and behavior correlations (Christodoulou et al., 2001; Perlstein et al., 2004), these studies reported recruitment of right lateral PFC regions during working memory task performance, consistent with our findings. Moreover, Perlstein et al. (2004) also reported a group by laterality interaction whereby increased working memory load was associated with increased activity in left lateral PFC for controls and right lateral PFC for TBI consistent with our data (Figure 2).

We were particularly interested in questions of functional connectivity as our TBI sample was carefully screened for evidence of focal brain pathology, providing us with a sample of patients with relatively "pure" DAI. Higher cognition is increasingly understood to be an emergent property of brain activity within large scale neural networks anchored by nodes within PFC (McIntosh, 1999; Grady et al., 2003). Given that working memory is disrupted following TBI (e.g., D'Esposito et al., 2006), we surmised that such networks subserving working memory might be particularly sensitive to DAI. Moreover, data from our first analysis suggested that compensatory functional recruitment of right PFC following TBI was replicated in controls, but only at the highest level of ED within working memory. Consistent with recent reports in healthy aging (Lustig et al., 2009), these data suggested that this pattern of right lateralized recruitment may reflect a pattern of task-dependent altered functional engagement rather than functional reorganization per se.

To test this hypothesis, we employed multivariate methods (combined bPLS and sPLS) to examine the functional connectivity and behavioral relevance of networks anchored by four PFC "seed" regions, identified in the bPLS analysis as being highly correlated with performance on our working memory tasks. The results were consistent with an altered functional engagement hypothesis. Lateral regions of PFC including aGFm bilaterally, left GFi and the border zone of right GFi and GFm, were functionally connected in both groups. Their behavioral relevance, however was differentially altered both by task demands and by brain injury (see Figure 4 for an overview of these results within PFC). Increasing either load or ED above baseline (Maintain 3) resulted in recruitment of right anterior PFC into the baseline left lateralized PFC network. Activity in this expanded network was only sufficient to support behavioral performance in controls. TBI patients required additional recruitment of right posterior lateral $\mathrm{PFC}$ to support performance at this level of task demand (Maintain 5, Alphabetize 3). Control performance was correlated with activity in this broader bilateral PFC network only when both ED and load increased (Alphabetize 5). Thus the stepwise pattern of behaviorally relevant functional recruitment observed in our controls as working memory demands increased was truncated for TBIs where right PFC brain-behavior correlation was observed with any increase in task demand.

When interpreting functional brain changes in neurological populations it is critical to consider the potential contribution of injury-related changes in the microvasculature, and thus BOLD signals, to observed group differences (see D'Esposito et al., 2003 for a review). We cannot directly rule out the impact of TBI-related vascular changes in our results. However, we observed robust lateralization and task-related differences in both brain and behavior correlations (Figure 2; and Figure A1 in Appendix) as well as task-dependent differences in patterns of network connectivity (Figure 3 ) between our groups. This pattern of group-wise, task-dependent changes in localized brain regions could not be explained by a more generalized account of altered BOLD signaling following TBI.

In summary, these results support the altered functional engagement hypothesis following TBI. Existing PFC nodes that are normally coactivated during working memory task performance are behaviorally relevant at an earlier stage of difficulty for TBI patients as compared to controls. This finding is inconsistent with the notion of reorganization whereby new network nodes not previously engaged by the task are related to task performance. This would not have been apparent had we not included a functional connectivity analysis (sPLS) to assess extant, but latent PFC networks. Without such analysis, brain-behavior correlations may be misinterpreted as reflecting reorganization when in fact they reflect altered functional engagement. It is important to note that the controls' behaviorally relevant left-lateralized network indicated frontoparietal connectivity classically associated with working memory (Postle, 2006), whereas the TBI patients' right-lateralized network also included midline posterior regions not present for controls, consistent with previous reports (Levine et al., 2006). As this paper focuses on functional changes within PFC related to executive control within working memory, we cannot rule out that the inclusion of these midline posterior regions in the TBI patients reflects functional reorganization. However, we did not examine this directly here.

In a review of activation functional imaging in TBI, Levine et al. (2006) concluded that brain activity is more widely dispersed and weaker in PFC relative to non-injured controls. However, they cautioned that confirmation of these functional brain changes in TBI would have to await studies of TBI subjects with more homogenous neuropathology, well-validated 


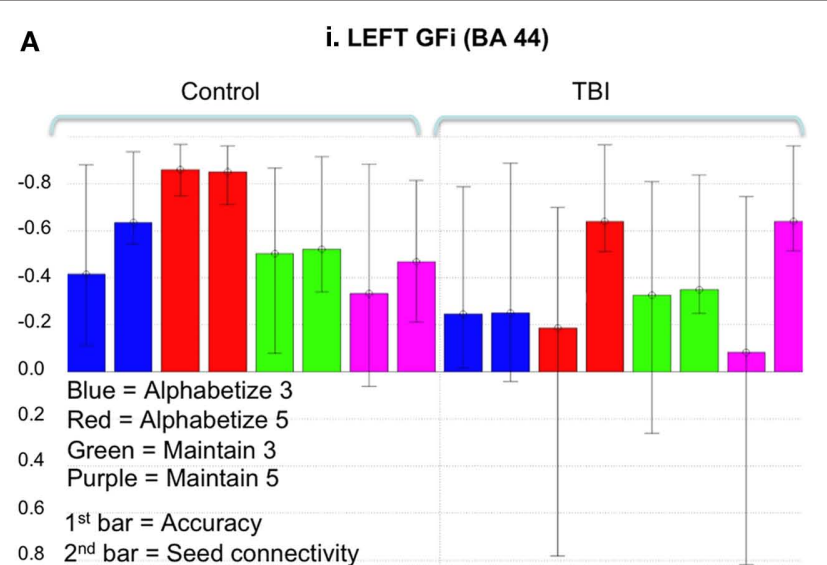

ii. LEFT GFm (BA 46)

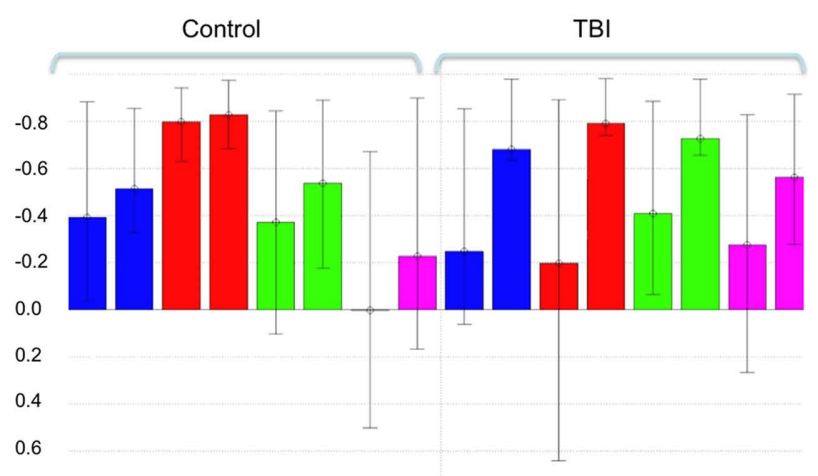

0.8
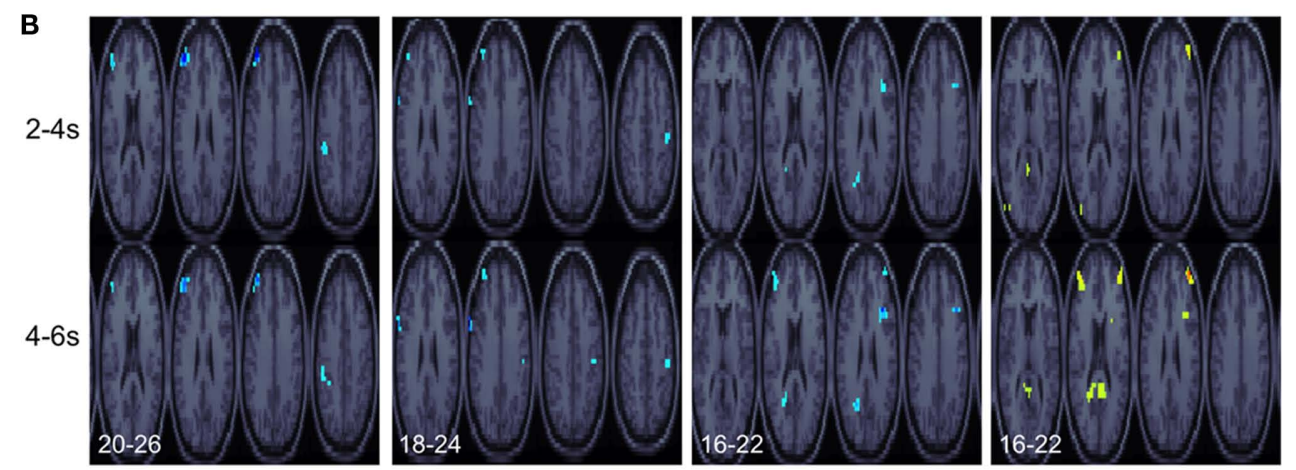

FIGURE 3 | (A) Behavioral and seed LVs (LV 1 all analyses) for the four PFC ROIs indicated in Figure 2. Legend in (Ai) applies to all histograms. For consistency of presentation, correlation values are reversed on the $x$-axis for (Ai-iii). Significant brain saliences for these LVs were negative (B), thus indicating a positive behavior and seed correlation. Error bars represent confidence intervals defined by the standard error of the bootstrap estimates (as in Figure 1). (B) BSRs for each of the LVs represented in (A). Slice coordinate in
Talairach space $(z)$ are indicated in bottom-left (step $=2$ ). Time lags (either $2-4$ or 4-6 s post stimulus onset) are indicated on the left of the images. BSR was set at $4\left(p<10^{-4}\right.$, corresponding approximately to a $\left.p<0.0001\right)$ and cluster size was $>5$ voxels for all images. As noted above, the sign of the correlation and voxel saliences was reversed between images (Ai-iv). This is reflected in the voxel color coding in (Aiv). However, this does not affect interpretation; all brain-behavior correlations were positive. experimental paradigms and matched behavioral performance. Our investigation meet these criteria and extend their conclusions in two important ways. First, we demonstrate that the pattern of spatially dispersed activity in TBI positively correlates with task performance - i.e., these functional brain changes in this population are compensatory. Secondly, the pattern of altered functional recruitment we observed in this sample of TBI participants mirrored that observed in healthy controls - but at lower levels of task challenge. In other words, TBI was associated with poor regulation of functional brain activity in response to increasing task demand.

The neuropathological profile of our patient sample is similar to that reported in studies of healthy aging where diffuse white matter pathology and generalized reductions in gray and white matter 


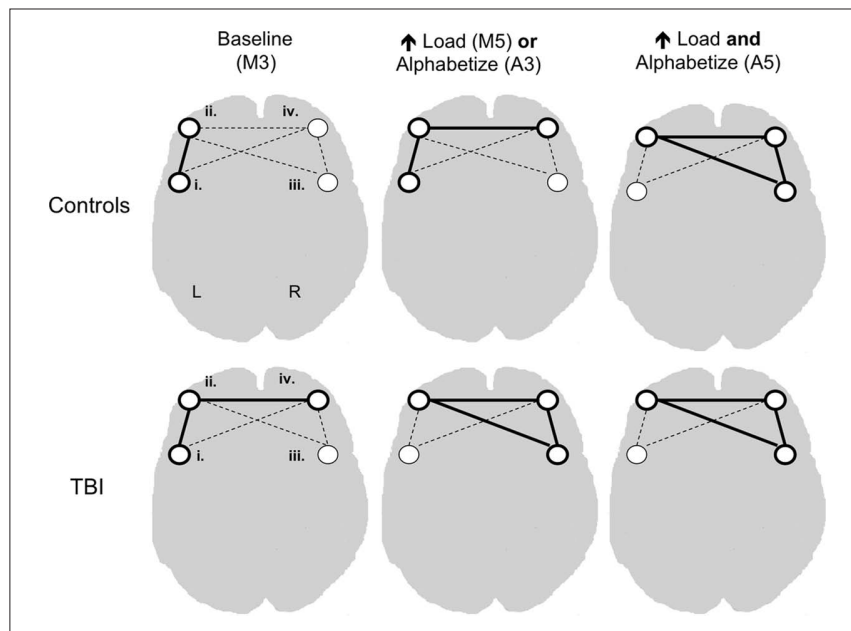

FIGURE 4 | Conceptual representation of combined behavioral and seed partial least squares analysis. Dashed lines connecting seed regions represent functional connectivity between these regions. Solid lines signify both functional connectivity and correlations between activity in the network and task accuracy. Roman numerals signify seed regions as defined in Figure 1.

\section{REFERENCES}

Cabeza, R., and Nyberg, L. (2000). Imaging cognition II: an empirical review of 275 PET and fMRI studies. J. Cogn. Neurosci. 12, 1-47.

Christodoulou, C., DeLuca, J., Ricker, J. H., Madigan, N. K., Bly, B. M., Lange, G., Kalnin, A. J., Liu, W.-C., Steffener, J., Diamond, B. J., and Ni, A.C. (2001). Functional magnetic resonance imaging of working memory impairment after traumatic brain injury. J. Neurol. Neurosurg. Psychiatry, 71, 161-168.

Cox, R. W., and Hyde, J. S. (1997). Software tools for analysis, and visualization of fMRI data. NMR Biomed. 10, 171-178.

Craik, F. I. M. (1986). "A functional account of age differences in memory," in Human Memory and Cognitive Capabilities, Mechanisms and Performances, eds F. Klix and H. Hagendorf (New York: Elsevier Science), 409-422.

Curtis, C. E., and D'Esposito, M. (2003). Persistent activity in the prefrontal cortex during working memory. Trends Cogn. Sci. 7, 415-423.

D’Esposito, M., Cooney, J.W., Gazzaley,A., Gibbs, S. E., and Postle, B. R. (2006). Is the prefrontal cortex necessary for delay task performance? Evidence from lesion and FMRI data. J. Int. Neuropsychol. Soc. 12, 248-260.

D'Esposito, M., Deouell, L. Y., and Gazzaley, A. (2003). Alterations in the bold fMRI signal with ageing and disease: a challenge for neuroimaging. Nat. Rev. Neurosci. 4, 863-872.

Dockree, P. M., Kelly, S. P., Roche, R. A. P., Hogan, M. J., Reilly, R. B., and
Robertson, I. H. (2004). Behavioural and physiological impairments of sustained attention after traumatic brain injury. Cogn. Brain Res. 20, 403-414.

Duncan, C. C., Kosmidis, M. H., and Mirsky, A. F. (2005). Closed head injury-related information processing deficits: an event-related potential analysis. Int. J. Psychophysiol. 58, 133-157.

Erickson, K. I., Colcombe, S. J., Wadhwa, R., Bherer, L., Peterson, M. S., Scalf, P. E., Kim, J. S., Alvarado, M., and Kramer,A.F.(2007). Training-induced plasticity in older adults: effects of training on hemispheric asymmetry. Neurobiol. Aging 28, 272-283.

Friston, K. J., Holmes, K. J., Worsley, J.-P., Poline, C. D., Frith, C. D., and Frackowiak, R. S. J. (1995). Statistical parametric maps in functional neuroimaging: a general linear approach. Hum. Brain Mapp. 2, 189-210.

Grady, C. L., McIntosh, A. R., Beig, S., Keightley, M.L., Burian, H., and Black, S.E. (2003). Evidence from functional neuroimaging of a compensatory prefrontal network in Alzheimer's disease. J. Neurosci. 23, 986-993.

Levine, B., Fujiwara, E., O'Connor, C., Richard, N., Kovacevic, N., Mandic, M., Restagno, A., Easdon, C., Robertson, I. H., Graham, S. J., Cheung, G. K., Gao, F. H., Schwartz, M. L., and Black, S. E. (2006). In vivo characterization of traumatic brain injury neuropathology with structural and functional neuroimaging. $J$. Neurotrauma 23, 1396-1411.

Levine, B., Kovacevic, N., Nica, I., Cheung, G., Schwartz, M. L., and Black, S. E.

have been observed (e.g., Tisserand et al., 2002; Raz et al., 2005). Interestingly, studies examining the functional neuroanatomy of working memory in healthy aging (e.g., Rypma and D'Esposito, 2000; Erickson et al., 2007; see Lustig et al., 2009 for a review), also report similar patterns of compensatory right prefrontal recruitment as we observed in our TBI sample. This convergence of neuropathology and functional recruitment patterns suggests that healthy aging may in part mimic DAI, at least with respect to the functional neuroanatomy of higher cognition.

Finally, we have previously reported that our TBI patients have evidence of DAI on neuroradiological report. Moreover, there were significant group-wise differences in cerebral white and gray matter in this TBI group relative to healthy age- and education-matched controls (Turner and Levine, 2008). An important next step in understanding the neural and behavioral sequelae of moderate to severe TBI will be to relate these structural brain changes to the pattern of functional brain changes reported here. Work is currently underway in our laboratory to examine this question using diffusion imaging techniques to characterize the structural integrity of white matter pathways in this population.

(2008). Toronto traumatic brain injury study: injury severity and quantified MRI. Neurology 70, 771-778.

Lustig, C., Shah, P., Seidler, R., and ReuterLorenz, P. (2009). Aging, training and the brain: a review and future directions. Neuropsychol. Rev. 19, 504-522.

McAllister, T. W., Saykin, A. J., Flashman, L. A., Sparling, M. B., Johnson, S. C., Guerin, S. J., Mamourian, A. C., Weaver, J. B., and Yanofsky, N. (1999). Brain activation during working memory 1 month after mild traumatic brain injury: a functional MRI study. Neurology 53, 1300-1308.

McIntosh, A. R. (1999). Mapping cognition to the brain through neural interactions. Memory 7, 523-548.

McIntosh, A. R., Bookstein, F. L., Haxby, J. V., and Grady, C. L. (1996). Spatial pattern analysis of functional brain images using partial least squares. Neuroimage 3, 143-157.

McIntosh, A. R., Chau, W. K., and Protzner,A. B. (2004). Spatiotemporal analysis of event-related fMRI data using partial least squares. Neuroimage 23, 764-775.

McIntosh, A. R., and Lobaugh, N. J. (2004). Partial least squares analysis of neuroimaging data: applications and advances. Neuroimage 23 (Suppl. 1), S250-S263.

Molteni, E., Baselli, G., Bianchi, A. M., Caffmi, M., Contint, D., Spinelli, L., Torricelli,A., Cerutti, S., and Cubeddu, R. (2009). Frontal brain activation during a working memory task: a time-domain fNIRS study. Prog. Biomed. Opt. Imaging Proc. SPIE 7161, Art. No. 71613 N.
Newsome, M. R., Scheibel, R. S., Seignourel, P. J., Steinberg, J. L., Troyanskaya, M., Li, X., and Levin, H. S. (2009). Effects of methylphenidate on working memory in traumatic brain injury: a preliminary fMRI investigation. Brain Imaging Behav. 3, 298-305.

Newsome, M. R., Scheibel, R. S., Steinberg, J. L., Troyanskaya, M., Sharma, R. G., Rauch, R. A., Li, X., and Levin, H. S. (2007). Working memory brain activation following severe traumatic brain injury. Cortex 43, 95-111.

Ogawa, S., Lee, T. M., Kay, A. R., and Tank, D. W. (1990). Brain magneticresonance-imaging with contrast dependent on blood oxygenation. Proc. Natl. Acad. Sci. U.S.A. 87, 9868-9872.

Paxton, J. L., Barch, D. M., Racine, C. A., and Braver, T.S. (2008). Cognitive control, goal maintenance, and prefrontal function in healthy aging. Cereb. Cortex 18, 1010-1028.

Perlstein, W. M., Cole, M. A., Demery, J. A., Seignourel, P. J., Dixit, N. K., Larson, M. J., and Briggs, R. W. (2004). Parametric manipulation of working memory load in traumatic brain injury: behavioral and neural correlates. J. Int. Neuropsychol. Soc. 10, 724-741.

Postle, B. R. (2006). Working memory as an emergent property of the mind and brain. Neuroscience 139, 23-38.

Postle, B. R., Berger, J. S., and D'Esposito, M. (1999). Functional neuroanatomical double dissociation of mnemonic and executive control processes 
contributing to working memory performance. Proc. Natl. Acad. Sci. U.S.A. 96, 12959-12964.

Povlishock, J. T., and Katz, D. I. (2005). Update of neuropathology and neurological recovery after traumatic brain injury. J. Head Trauma Rehabil. 20, 76-94.

Raz, N.,Lindenberger, U., Rodrigue, K.M., Kennedy, K. M., Head, D., Williamson, A., Dahle, C., Gerstorf, D., and Acker, J.D. (2005). Regional brain changes in aging healthy adults: general trends, individual differences and modifiers. Cereb. Cortex 15, 1676-1689.

Rypma, B., and D’Esposito, M. (2000). Isolating the neural mechanisms of age-related changes in human working memory. Nat. Neurosci. 3 , 509-515.

Rypma, B., Prabhakaran, V., Desmond, J. E., Glover, G. H., and Gabrieli, J. D.
(1999). Load-dependent roles of frontal brain regions in the maintenance of working memory. Neuroimage 9 , 216-226.

Stuss, D. T., Pogue, J., Buckle, L., and Bondar, J. (1994). Characterization of stability of performance in patients with traumatic brain injury: variability and consistency on reaction time tests. Neuropsychology 8, 316-324.

Stuss, D. T., Stethem, L. L., Hugenholtz, H., Picton, T., Pivik, J., and Richard, M. T. (1989). Reaction time after traumatic brain injury: fatigue, divided and focused attention, and consistency of performance. J. Neurol. Neurosurg Psychiatry 52, 742-748.

Teasdale, G., and Jennett, B. (1974). Assessment of coma and impaired consciousness. A practical scale. Lancet 2, 81-84.
Tisserand, D. J., Pruessner, J. C., Sanz Arigita, E. J., van Boxtel, M. P. J., Evans, A. C., Jolles, J., and Uylings, H. B. M. (2002). Regional frontal cortical volumes decrease differentially in aging: an MRI study to compare volumetric approaches and voxel-based morphometry. Neuroimage 17, 657-669.

Turner, G. R., and Levine, B. (2008). Augmented neural activity during executive control processing following diffuse axonal injury. Neurology $71,812-818$.

Wager, T. D., and Smith, E. E. (2003). Neuroimaging studies of working memory: A meta-analysis. Cognitive, Affective and Behavioral Neuroscience, 3, 255-274.

Conflict of Interest Statement: The authors declare that the research was conducted in the absence of any commercial or financial relationships that could be construed as a potential conflict of interest.

Received: 06 August 2010; accepted: 08 February 2011; published online: 24 February 2011.

Citation: Turner GR, McIntosh AR and Levine B (2011) Prefrontal compensatory engagement in TBI is due to altered functional engagement of existing networks and not functional reorganization. Front. Syst. Neurosci. 5:9. doi: 10.3389/ fnsys.2011.00009

Copyright (c) 2011 Turner, McIntosh and Levine. This is an open-access article subject to an exclusive license agreement between the authors and Frontiers Media $S A$, which permits unrestricted use, distribution, and reproduction in any medium, provided the original authors and source are credited. 


\section{APPENDIX}

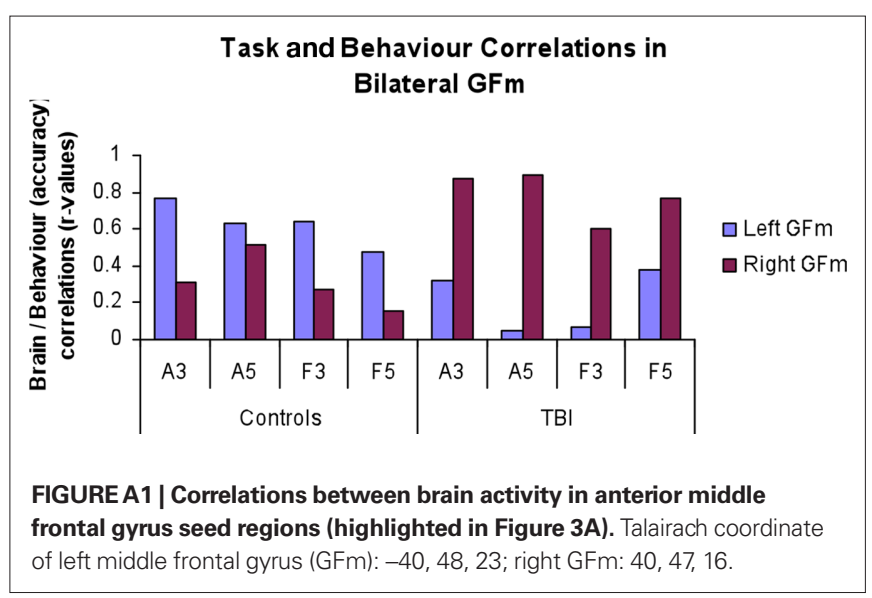

